\title{
Non-Cardiogenic Pulmonary Edema Complicating Electroconvulsive Therapy: Short Review of the Pathophysiology and Diagnostic Approach
}

\author{
Janaki R. Manne, MD; Yusuf Kasirye, MD; Narendranath Epperla, MD; and
} Romel J. Garcia-Montilla, MD, PhD, MSc

\begin{abstract}
Acute pulmonary edema complicating electroconvulsive therapy is an extremely uncommon event that has rarely been described in the literature. Different theories, including one suggesting a cardiogenic component, have been proposed to explain its genesis. The present report describes a classic presentation of this condition with review of its potential mechanisms and diagnostic approach. After successful completion of a session of electroconvulsive therapy, a 42-year-old woman with major depressive disorder developed acute systemic high blood pressure, shortness of breath, and hemoptysis. A chest radiograph demonstrated diffuse bilateral pulmonary infiltrates. Initially cardiogenic pulmonary edema was presumed, but an extensive diagnostic work-up demonstrated normal systolic and diastolic left ventricular function, and with only supportive measures, a complete clinical and radiographic recovery was achieved within 48 hours. The present case does not support any cardiogenic mechanism in the genesis of this condition.
\end{abstract}

Keywords: Airway obstruction; Electroconvulsive therapy; Pulmonary edema

Corresponding Author:

Dr. Romel Garcia-Montilla

Department of Hospital Medicine

Marshfield Clinic

1000 North Oak Ave

Marshfield, WI 54449 USA

Tel: (7I5) 387-5537

Fax: (715) 389-5757

Email: garcia-montilla.romel@marshfieldclinic.org

Received: June 22, 2011

Revised: September 13, 201|

Accepted: September 21, 2011

doi: $10.3121 / \mathrm{cmr} .2011 .1030$
$\mathrm{T}$

wo types of pulmonary edema occur in clinical medicine: cardiogenic pulmonary edema (or hydrostatic pulmonary edema) and noncardiogenic pulmonary edema, better known as acute lung injury or acute respiratory distress syndrome (ARDS). Their clinical manifestations are very similar, so the differentiation between them, based only on clinical grounds, may be very difficult, and knowing the precise etiology of the episode of acute pulmonary edema has major implications in the treatment plan. Cardiogenic pulmonary edema is usually due to systolic and/or diastolic left ventricular dysfunction and is typically treated with diuretics, nitrates, and afterload reduction medications such as angiotensin converting enzyme inhibitors, although some cases also require coronary revascularization. Noncardiogenic pulmonary edema is usually secondary to a more systemic severe medical or surgical pathology that triggers the event, and the treatment should be directed to treat that pathology. Oxygen supplementation in the form of mechanical ventilation (invasive or non-invasive) is always required. A rapid diagnosis of the cause of the episode of acute pulmonary edema facilitates a timely and appropriate therapeutic intervention.

Electroconvulsive therapy (ECT) is recognized as a well-established, highlyeffective, safe psychiatric treatment. It is estimated that 100,000 patients per year receive a course of ECT across the United States, with an average of 8 to 10 treatments per course of therapy. Severe complications are very infrequent and generally reported as transitory cardiac arrhythmias and severe transitory 


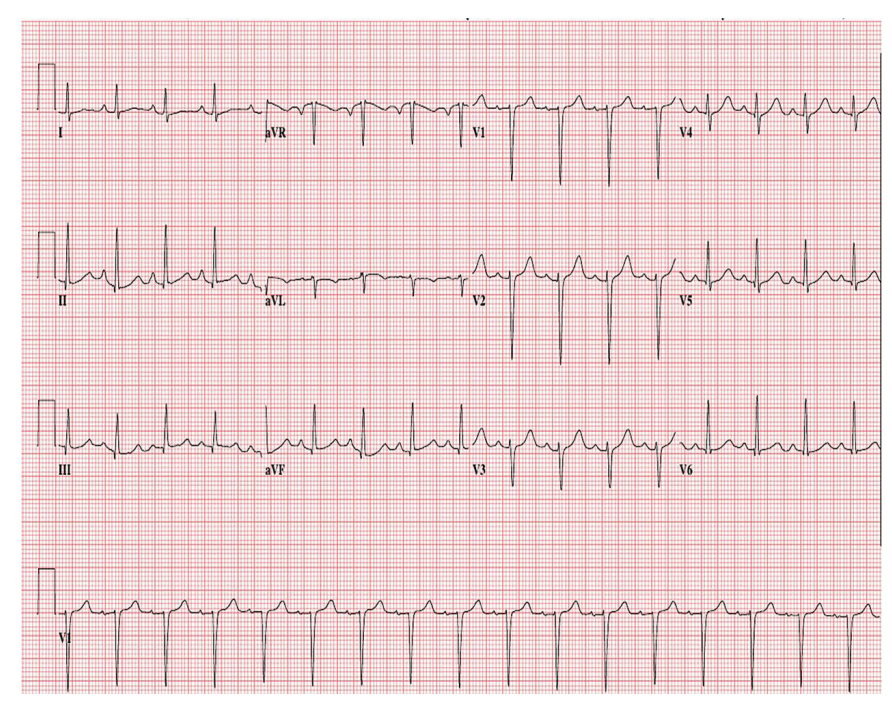

Figure 1. Electrocardiogram showing sinus tachycardia.

hypertension without immediate consequences. ${ }^{1,2}$ Acute pulmonary edema after ECT is an unpredictable and extremely uncommon condition that has rarely been reported before; even extensive reviews about uncommon complications and serious sides effects of ECT do not mention it. ${ }^{3,4}$ To the best of our knowledge, only nine cases have been described in the literature since 1982. Most of these previous reports have suggested a cardiogenic component as one mechanism in the genesis of this condition; however, despite extensive diagnostic workups, none of them have included a brain natriuretic peptide (BNP) level determination as a valuable tool in the evaluation of patients with acute pulmonary edema, based on its well-recognized power to differentiate between cardiogenic and noncardiogenic pulmonary edema. We present a case of acute pulmonary edema complicating ECT, and we review the different potential pathogenetic mechanisms involved, where a complete work up including BNP does not support any cardiogenic component in its genesis.

\section{Case Report}

A 42-year-old, non-obese, white woman with a past medical history significant only for severe major depressive disorder presented for her third course of ECT for the treatment of her depression (she had previously received two courses of ECT, within the prior three years without complications). Following the successful completion of her ECT session, after spontaneous respiration, normal awakening, and full orientation were resumed, she developed sinus tachycardia, tachypnea, and severe blood pressure elevation. She suddenly became very agitated with laborious breathing and coughing up an abundant amount of pink frothy sputum with streaks of blood. Her hemoglobin oxygen saturation decreased to the middle $60 \%$ range. An initial diagnosis of acute cardiogenic pulmonary edema was presumed.

At the time of our evaluation, the patient was agitated with blood pressure $240 / 140 \mathrm{mmHg}$, respirations 40 per minute, and heart rate 140 beats per minute. Physical examination was remarkable only for bilaterally diffuse, easily audible, coarse crackles. An electrocardiogram showed sinus tachycardia but no other abnormalities (figure 1). Chest radiograph demonstrated a cardiac silhouette appearing normal in size, shape, and contour, with extensive bilateral fluffy opacities and air bronchograms, with a non-focal consolidation pattern suggestive of acute respiratory distress syndrome (ARDS) (figure 2). Laboratory studies revealed a BNP level of $39 \mathrm{pg} / \mathrm{mL}$ (normal range: $5-100 \mathrm{pg} / \mathrm{mL}$ ). Three consecutive troponin I readings were negative. Echocardiogram was normal, reporting no wall motion abnormalities, with an ejection fraction of $65 \%$ and no signs of diastolic dysfunction. Pheochromocytoma screening with normetanephrine and metanephrine levels was negative. Renal ultrasound showed normal kidneys and adrenal glands. A complete blood cell count and a basic metabolic panel were also all within normal limits.

The patient received acutely, in a rapid sequence, intravenous labetalol (20 mg), morphine (3 mg), and furosemide (40 mg) and subsequently developed hypotension with a blood pressure of $86 / 56 \mathrm{mmHg}$; noninvasive mechanical ventilation with bilevel positive airway pressure (BiPAP) and 100\% oxygen was started. She was transferred to the critical care unit for further monitoring and management, where no additional medications were administered. A few hours later all her vital signs were stable, the BiPAP was stopped, she was weaned to room air without difficulty and was transferred out of the critical care unit to a regular medical floor. The

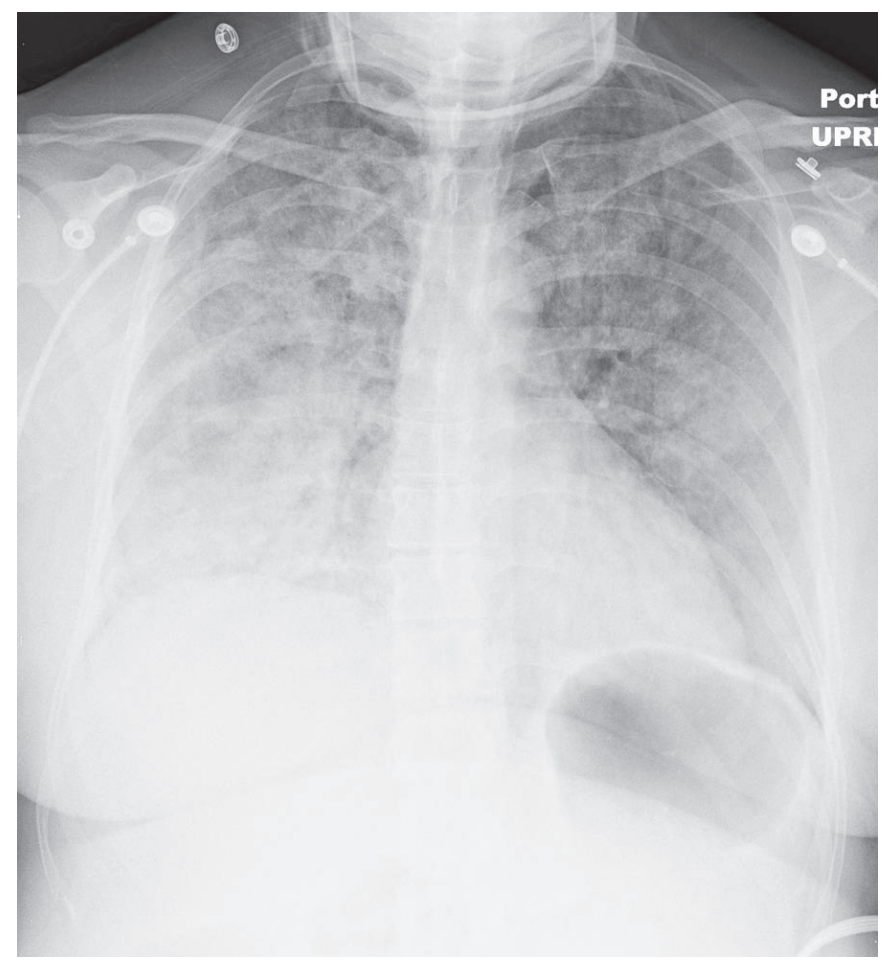

Figure 2. Chest radiograph during the episode of dyspnea. Cardiac silhouette normal in size, shape and contour, extensive bilateral opacities more pronounced on the bases with air bronchograms can be observed. 


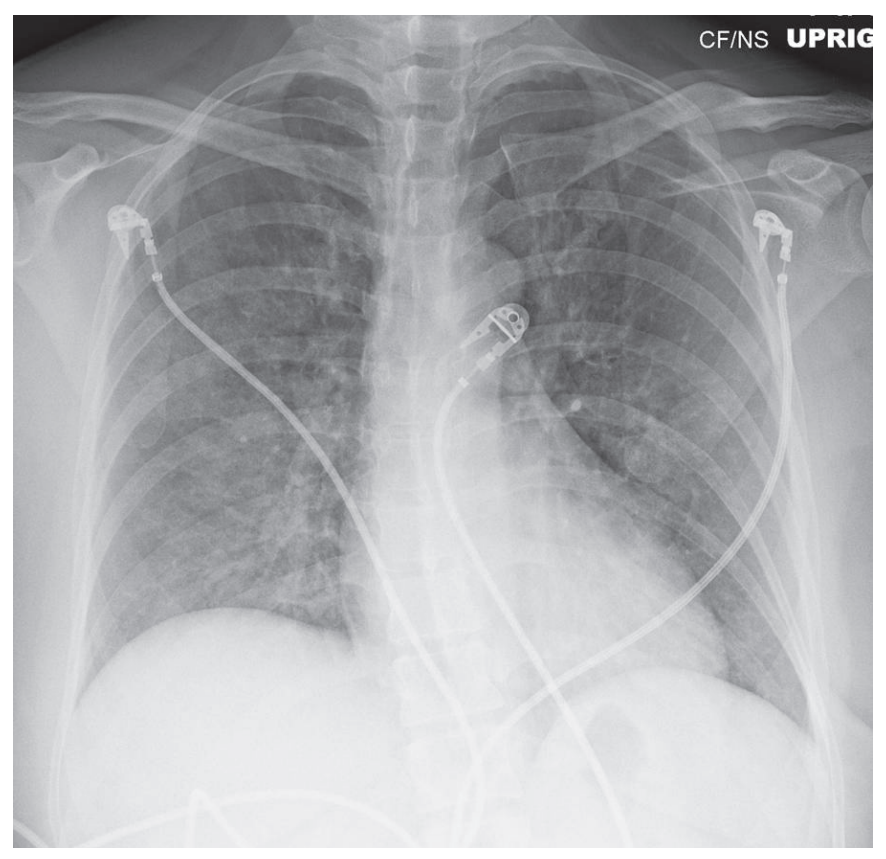

Figure 3. Chest radiograph at 24 hours.

control chest radiographs at 24 and 48 hours showed a very fast and dramatic improvement in the radiographic findings (figures 3 and 4).

\section{Discussion}

Cardiovascular reaction to ECT involves a strong autonomic response. Animal models and clinical observations suggest that overstimulation or damage of specific neuron groups located in the hypothalamus, brainstem, and cervical spine are capable of generating an imbalance in the peripheral vascular and pulmonary vascular autonomic nervous systems. This imbalance may produce a clinical condition known as "catecholamine storm" that is characterized by severe but short lasting (only minutes) changes in systolic and diastolic blood pressure, heart rate, and pulmonary pressures, and is able to create a mismatch of the Starling forces in the pulmonary vascular bed generating edema. ${ }^{5}$ The overactivation of the sympathetic nerve endings leads to peripheral vasoconstriction with subsequent increase in systemic blood pressure and augmentation of central blood volume. These changes are followed by the constriction of the pulmonary veins, producing an increase in the pulmonary capillary hydrostatic pressure with subsequent elevation in the pulmonary pressure beyond the physiological tolerance (about $39 \mathrm{mmHg}$ ) of the alveolar-capillary membrane with the resultant damage (stress failure) of all layers of the blood-gas barrier, causing leakage of protein-rich fluid and blood into the interstitium and intra-alveolar space, resulting in the typical picture of acute pulmonary edema. ${ }^{5,6}$ All these pathophysiological changes happen in the presence of normal left atrial pressure (normal pulmonary wedge pressure), indicating a non-cardiogenic etiology. $5,7,8$
The exaggerated autonomic sympathetic response associated with ECT may also produce an isolated sudden increase of the systolic blood pressure by more than $40 \%$ above its baseline. ${ }^{9}$ This acute elevation of blood pressure may induce a sudden and transient left ventricular diastolic dysfunction, producing pulmonary edema in the same way as in those cases of hypertension-induced cardiogenic pulmonary edema. ${ }^{10}$

It is well documented that ECT increases myocardial oxygen demand, and left ventricular wall motion abnormalities and electrocardiographic changes suggestive of myocardial ischemia have been described. ${ }^{11}$ These phenomena may produce a transient left ventricular dysfunction leading to acute pulmonary edema as that observed during an acute coronary syndrome.

Another theory implicated in the genesis of post-ECT pulmonary edema is a severely increased negative intrapulmonary pressure. This hypothesis suggests that forced inspiration against a closed glottis during ECT creates a very high negative intrathoracic pressure, promoting an increased venous return to the thorax, with the consequent transudation of fluid to pulmonary interstitium and alveoli, a condition known as post-obstructive pulmonary edema. ${ }^{12}$ The clinical manifestations would not become evident until after relief of the airway obstruction. ${ }^{13}$ In our case, the manifestations developed several minutes after a full recovery was achieved, and the modern practice of total muscular paralysis (our patient was pre-medicated with thiopental $375 \mathrm{mg}$ and succinylcholine $140 \mathrm{mg}$ ) with a secured airway during ECT (both goals achieved in this case) make this particular mechanism of genesis very unlikely.

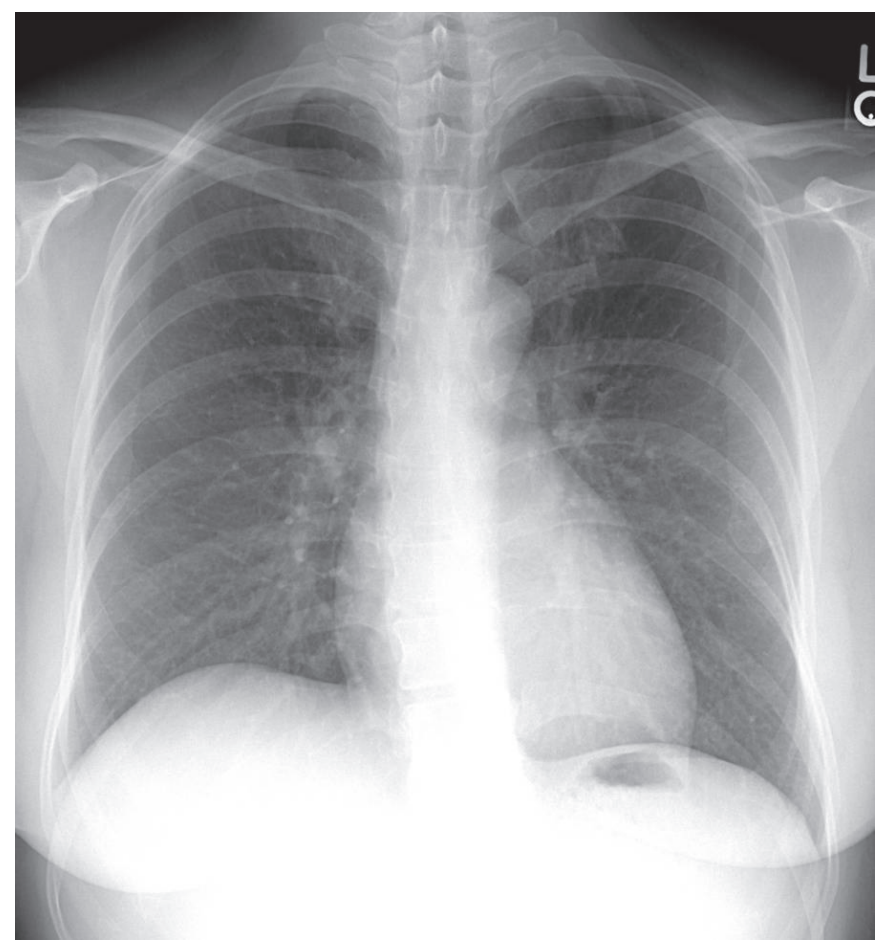

Figure 4. Chest radiograph at 48 hours. An absolutely normal study. 
As previously mentioned, acute pulmonary edema after ECT may have different potential pathophysiologic mechanisms, and a multifactorial model may be the most suitable explanation for its genesis; however, in our case, a normal cardiac workup, a chest radiograph compatible with ARDS, and particularly a strikingly normal BNP level, do not support a cardiogenic mechanism. It is routine to use BNP level determinations in the diagnostic evaluation of patients with acute pulmonary edema. ${ }^{14}$ This protein is secreted by the ventricles in response to an increase in left ventricular end diastolic pressure (LVEDP), and its levels correlate positively with LVEDP and the New York Heart Association functional class. ${ }^{15,16}$ In the absence of mitral valvulopathy (ie, mitral stenosis), which may produce left atrial hypertension with a normal left ventricular function, an elevated LVEDP with its retrograde transmission to the pulmonary capillaries is a sine qua non condition for the development of cardiogenic pulmonary edema, irrespective of whether this elevation is due to systolic or diastolic left ventricular dysfunction. In other words, the worse the left ventricular dysfunction, the higher the LVEDP, and consequently, the higher the BNP level. ${ }^{16}$ In 2004, the BNP Consensus Panel established that values below $100 \mathrm{pg} / \mathrm{mL}$ indicate that pulmonary edema is not cardiogenic, with a negative predictive value of more than $90 \% .{ }^{17}$ In a recent study done in patients requiring admission to a critical care unit, when measured between the first three hours after the onset of acute pulmonary edema, a BNP level below $250 \mathrm{pg} / \mathrm{mL}$ supports the diagnosis of acute lung injury rather than cardiogenic pulmonary edema with a specificity of $87 \% .^{18}$

Based on the information mentioned above, and considering the clinical presentation and subsequent rapid resolution of the symptoms in our patient, a final diagnosis of ECT-induced acute neurogenic pulmonary edema (NPE) was established. Neurogenic pulmonary edema is an unusual form of noncardiogenic pulmonary edema; it is defined as an acute increase in pulmonary interstitial and alveolar fluid occurring shortly after a central nervous system (CNS) insult without any alternative pre-existing or co-existing pathology to explain it. It has been reported in various conditions involving the CNS, including ECT, although epileptic seizures, head trauma, and cerebral hemorrhage are by far the primary causes. ${ }^{19-21}$ In patients with the most severe forms of CNS lesions, NPE may have a morbidity and mortality of $50 \%$ and $7 \%$, respectively. ${ }^{5}$ Although this entity has been recognized since 1908 , its pathophysiology is still poorly understood. ${ }^{22}$

Neurogenic pulmonary edema is unpredictable and presents within minutes of the CNS insult. Sudden onset of dyspnea and hemoptysis are the most common manifestations. Physical examination reveals tachypnea, tachycardia, and pulmonary crackles. Chest radiography typically shows a normal sized heart with diffuse bilateral alveolar filling resembling ARDS. Hemodynamic measurements such as high blood pressure, increased pulmonary pressure, and tachycardia are usually normal by the time NPE is diagnosed, mainly due to the very early occurrence and short duration of these manifestations. $5,23,24$
Definitive diagnosis of NPE is very difficult because of the nonspecific nature of clinical signs and the lack of a specific test. Similar to our patient, most cases are initially classified as cardiogenic pulmonary edema, and the treatment is initiated based on this assumption, so the diagnosis is largely based upon the occurrence of acute pulmonary edema in the appropriate clinical setting after ruling out other causes of the manifestations, mainly aspiration pneumonia (a frequent complication of patients with a neurological insult) and congestive heart failure. Neurogenic pulmonary edema tends to develop and resolve more rapidly (lasts hours) than aspiration pneumonia (lasts days), and the presence of a high white blood cell count and fever with more focal infiltrates on chest radiograph favor the latter. History of heart disease and a positive cardiac workup, including an elevated BNP, support congestive heart failure.

The outcome of patients with NPE is determined by the primary pathology, and any specific treatment must be focused on the underlying disorder. Neurogenic pulmonary edema itself is managed in a supportive fashion with most episodes resolving within 48 to 72 hours. Supplemental oxygen, the most valuable modality of therapeutic interventions, is always required, sometimes in the form of invasive mechanical ventilation. ${ }^{5,24} \mathrm{~A}$ variety of medications have been used to treat this condition, but their efficacy and safety are not firmly established mainly because of the small number of patients treated, the nonrandomized design of the studies, and the fact that NPE is usually a self-limited condition. Several agents such as alpha-adrenergic antagonists, beta-adrenergic blockers, dobutamine, and chlorpromazine are advocated by some, although no approach to the management of such patients is known to be superior. Cardiovascular agents, such as alpha blockers (ie, phentolamine) and beta blockers (ie, labetalol) are directed at blunting the exaggerated autonomic system responses and are used in situations where the goal is to lower the systemic blood pressure from dangerously high levels. ${ }^{23-25}$

In contrast to acute cardiogenic pulmonary edema where the use of loop diuretics (ie, furosemide) is justified based on a pathological state where preload and left atrial pressure (pulmonary wedge pressure) are increased, ${ }^{26,27}$ the evidence available about the pathogenesis and treatment of pure NPE where those parameters are normal ${ }^{5,7,8,24}$ does not support their use. ${ }^{5,24}$ Loop diuretics have a strong direct vascular action characterized by a prompt (as soon as 5 minutes after IV administration) and sometimes abrupt fall in mean pulmonary pressure, left atrial pressure, cardiac output, and arterial blood pressure irrespective of their diuretic effects, which may take up to 60 minutes to generate a clinically significant diuresis; ; $^{28}$ unrelated to their diuretic effect is the reduction in lung water content which takes several hours to be of clinical relevance. ${ }^{31}$ These observations indicate that the rapid symptomatic improvement, observed within minutes after the intravenous administration of loop diuretics in acute cardiogenic pulmonary edema, is not related to the diuretics, but rather to 
their vasodilator properties. ${ }^{28-31}$ In NPE, the pharmacological effects of this class of medications are additive to the effects of other cardiovascular agents used in its treatment (ie, beta and alpha blockers), increasing the risk of hypotension without addressing the root of this condition, which is the transient non-cardiogenic stress failure of the blood-gas barrier. ${ }^{5,6,8,24}$ In the treatment of presumed NPE, the loop diuretics are not indicated as first line agents and should be used exclusively to minimize a demonstrable cardiac component complicating the clinical picture, using the lowest possible effective dose and only if the systemic blood pressure, the circulating blood volume, and cerebral perfusion pressure can be maintained. ${ }^{24}$ Under the circumstances surrounding the development of NPE, extreme care must be taken to avoid systemic hypotension, as in the present case, with the consequently potential brain hypoperfusion in a clinical context where the cerebral circulatory autoregulation is probably already impaired.

\section{Conclusion}

Neurogenic pulmonary edema complicating ECT is an extremely rare condition with a poorly understood pathophysiology. Its diagnosis is one of exclusion but must be always considered in a patient receiving ECT who develops acute respiratory failure. Its treatment is mainly supportive in the form of mechanical ventilation (invasive or non-invasive) with positive end expiratory pressure. No medication is needed except for the control of excessively high blood pressure, avoiding hypotension. All physicians involved in the care of patients receiving ECT should be aware of this complication and understand its particular diagnosis and therapeutic approach in order to provide a timely and individualized treatment. The present case does not support a cardiogenic component in this condition, leaving a pure neurogenic mechanism as the most likely explanation for its genesis.

\section{Acknowledgments}

The authors wish to thank the Marshfield Clinic Research Foundation's Office of Scientific Writing and Publication for editorial assistance in the preparation of this manuscript.

\section{References}

1. Welch CA, Drop LJ. Cardiovascular effects of ECT. Convuls Ther 1989; 5:35-43.

2. Zielinski RJ, Roose SP, Devanand DP, Woodring S, Sackeim HA. Cardiovascular complications of ECT in depressed patients with cardiac disease. Am J Psychiatry 1993; 150:904-909.

3. Datto CJ. Side effects of electroconvulsive therapy. Depress Anxiety 2000; 12:130-134.

4. Cristancho MA, Alici Y, Augoustides JG, O’Reardon JP. Uncommon but serious complications associated with electroconvulsive therapy: recognition and management for the clinician. Curr Psychiatry Rep 2008; 10:474-480.

5. Sedý J, Zicha J, Kunes J, Jendelová P, Syková E. Mechanisms of neurogenic pulmonary edema development. Physiol Res 2008; 57:499-506.

6. Hachenberg T, Rettig R. Stress failure of the blood-gas barrier. Curr Opin Anaesthesiol 1998; 11:37-44.
7. Hoff JT, Nishimura M, Garcia-Uria J, Miranda S. Experimental neurogenic pulmonary edema. Part 1: The role of systemic hypertension. J Neurosurg 1981; 54:627-631.

8. Garcia-Uria J, Hoff JT, Miranda S, Nishimura M. Experimental neurogenic pulmonary edema Part 2: The role of cardiopulmonary pressure change. J Neurosurg 1981; 54:632-636.

9. Rumi DO, Solimene MC, Takada JY, Grupi CJ, Giorgi DM, Rigonatti SP, Luz PL, Ramires JA. Electrocardiographic and blood pressure alterations during electroconvulsive therapy in young adults. Arq Bras Cardiol 2002; 79:149-160.

10. Gandhi SK, Powers JC, Nomeir AM, Fowle K, Kitzman DW, Rankin KM, Little WC. The pathogenesis of acute pulmonary edema associated with hypertension. $\mathrm{N}$ Engl $\mathrm{J}$ Med 2001; 344:17-22.

11. Messina AG, Paranicas M, Katz B, Markowitz J, Yao FS, Devereux RB. Effect of electroconvulsive therapy on the electrocardiogram and echocardiogram. Anesth Analg 1992; 75:511-514.

12. Udeshi A, Cantie SM, Pierre E. Postobstructive pulmonary edema. J Crit Care 2010; 25):508.e1-5.

13. Myers CL, Gopalka A, Glick D, Goldman MB, Dinwiddie SH. A case of negative-pressure pulmonary edema after electroconvulsive therapy. J ECT 2007; 23:281-283.

14. Ware LB, Matthay MA. Clinical practice. Acute pulmonary edema. N Engl J Med 2005; 353:2788-2796.

15. Kazanegra R, Cheng V, Garcia A, Krishnaswamy P, Gardetto N, Clopton P, Maisel A. A rapid test for B-type natriuretic peptide correlates with falling wedge pressures in patients treated for decompensated heart failure: a pilot study. J Card Fail 2001; 7:21-29.

16. Maisel AS, Krishnaswamy P, Nowak RM, McCord J, Hollander JE, Duc P, Omland T, Storrow AB, Abraham WT, Wu AH, Clopton P, Steg PG, Westheim A, Knudsen CW, Perez A, Kazanegra R, Herrmann HC, McCullough PA; Breathing Not Properly Multinational Study Investigators. Rapid measurement of B-type natriuretic peptide in the emergency diagnosis of heart failure. N Engl J Med 2002; 347:161-167.

17. Silver MA, Maisel A, Yancy CW, McCullough PA, Burnett JC Jr, Francis GS, Mehra MR, Peacock WF 4th, Fonarow G, Gibler WB, Morrow DA, Hollander J; BNP Consensus Panel. BNP Consensus Panel 2004: A clinical approach for the diagnostic, prognostic, screening, treatment monitoring, and therapeutic roles of natriuretic peptides in cardiovascular diseases: Congest Heart Fail 2004; 10:1-30.

18. Rana R, Vlahakis NE, Daniels CE, Jaffe AS, Klee GG, Hubmayr RD, Gajic O. B-Type natriuretic peptide in the assessment of acute lung injury and cardiogenic pulmonary edema. Crit Care Med 2006; 34:1941-1946.

19. Colice GL, Matthay MA, Bass E, Matthay RA. Neurogenic pulmonary edema. Am Rev Respir Dis 1984; 130:941-948.

20. Price JW, Price JR, Perry TL. Excessive hypertension and pulmonary edema after electroconvulsive therapy. J ECT. $2005 ; 21: 174-177$.

21. Wayne SL, O’Donovan CA, McCall WV, Link K. Postictal neurogenic pulmonary edema: experience from an ECT model. Convuls Ther 1997; 13:181-184.

22. Shanahan WT. Acute pulmonary edema as a complication of epileptic seizures. New York Med 1908; 87:54.

23. Fontes RB, Aguiar PH, Zanetti MV, Andrade F, Mandel M, Teixeira MJ. Acute neurogenic pulmonary edema: case reports and literature review. J Neurosurg Anesthesiol 2003; $15: 144-150$

24. Baumann A, Audibert G, McDonnell J, Mertes PM. Neurogenic pulmonary edema. Acta Anaesthesiol Scand 2007; 51:447-455.

25. Sargent $P$, Reeves J. Pulmonary edema after electroconvulsive therapy. J ECT 2008; 24:283-285. 
26. Cotter G, Moshkovitz Y, Milovanov O, Salah A, Blatt A, Krakover R, Vered Z, Kaluski E. Acute heart failure: a novel approach to its pathogenesis and treatment. Eur J Heart Fail. 2002 Jun; 4(3):227-34.

27. Nieminen MS, Böhm M, Cowie MR, Drexler H, Filippatos GS, Jondeau G, Hasin Y, Lopez-Sendon J, Mebazaa A, Metra M, Rhodes A, Swedberg K, Priori SG, Garcia MA, Blanc JJ, Budaj A, Cowie MR, Dean V, Deckers J, Burgos EF, Lekakis J, Lindahl B, Mazzotta G, Morais J, Oto A, Smiseth OA, Garcia MA, Dickstein K, Albuquerque A, Conthe P, CrespoLeiro M, Ferrari R, Follath F, Gavazzi A, Janssens U, Komajda M, Morais J, Moreno R, Singer M, Singh S, Tendera M, Thygesen K; ESC Committe for Practice Guideline (CPG). Executive summary of the guidelines on the diagnosis and treatment of acute heart failure: the Task Force on Acute Heart Failure of the European Society of Cardiology. Eur Heart J 2005; 26:384-416.

28. Dikshit K, Vyden JK, Forrester JS, Chatterjee K, Prakash R, Swan HJ. Renal and extrarenal hemodynamic effects of furosemide in congestive heart failure after acute myocardial infarction. N Engl J Med 1973; 288:1087-1090.

29. Dormans TP, Pickkers P, Russel FG, Smits P. Vascular effects of loop diuretics. Cardiovasc Res 1996; 32:988-997.

30. Pickkers P, Dormans TP, Russel FG, Hughes AD, Thien T, Schaper N, Smits P. Direct vascular effects of furosemide in humans. Circulation 1997; 96:1847-1852.

31. Biddle TL, Yu PN. Effect of furosemide on hemodynamics and lung water in acute pulmonary edema secondary to myocardial infarction. Am J Cardiol 1979; 43:86-90.

\section{Author Affiliations}

Janaki R. Manne, MD*; Yusuf Kasirye, MD'; Narendranath Epperla, MD*; Romel J. Garcia-Montilla, MD, PhD, MSc*

*Department of Hospital Medicine, Marshfield Clinic, Marshfield, WI 54449 USA

†Department of Internal Medicine, Marshfield Clinic, Park Falls, WI 54552 USA

*Department of Internal Medicine, Marshfield Clinic, Marshfield, WI 54449 USA 